\title{
Approaches to Videogame Discourse
}

\author{
Bad Language and Bro-up Cooperation
}

\section{Chapter 6:}

\section{Bad Language and Bro-up Cooperation in Co-sit Gaming}

\section{Astrid Ensslin and John Finnegan}

\section{Introduction}

This chapter examines the quasi-paradoxical relationship between cooperative and impolite verbal behavior in co-situated ("co-sit") player communication. In doing so, it takes a broad paratextual, interactional approach, locating itself in the overlapping territory between videogame textuality research (see Part III of this volume), and verbal and multimodal player interaction. More specifically, we seek to investigate the role of BLE ("Bad Language Expressions," see McEnery 2006) as part of the discourses of cool and fun (Ensslin 2012) based on participants' use of swear words and other types of BLE, as well as their own assessment of the pragmatic functions and psychological and social motivations of these overused structures. This will be juxtaposed with an examination of the distinctly cooperative, mutually supportive and ultimately polite 'bro-up' behavior displayed by co-sit players to secure and maintain bonding and cooperation. The data is methodologically triangulated from transcribed and BLEannotated video/audio recordings and semi-structured paired interviews. We will show that the metaludic and metacommunicative information garnered from the interviews lifts the appearance of paradoxical behavior and instead refigures bad language as face-saving and ultimately prosocial tool in player communication. 


\section{Co-sit interaction as paratext}

Research into videogame paratexts has proliferated in the past five years or so, triggered partly by Mia Consalvo's seminal book, Cheating: Gaining Advantage in Videogames (2009), which first considered paratextual media (industries) such as game magazines, guides and walkthroughs, and how they blur the boundaries between game world and player world. Paratexts, according to Genette (1991), are "verbal and other productions" "beyond the naked state" of the text (Genette 1991: 261). Elements like the author's name, preface, illustrations, blurbs, interviews and reviews, "surround or extend" (Genette 1991:261) the core text of a book, which was Genette's medial focus. Clearly, however, no matter the medium, in contemporary participatory, fan culture, the text itself becomes the material starting point for multi-layered user interactions and communications, from debates in online discussion fora to cosplay (costume play) conventions and oral after-school chit-chat. It may thus be argued that, without the paratext, the text itself cannot come into being and that, in modern transmedia, "we can only approach texts through paratexts" (Gray 2010: 25). Games are an extreme case in point as their narratives are emergent and experiential (Aylett 1999, Jenkins 2004) and do not come into being without the player's "autotelic enactment" (Ryan 2004: 348), i.e. their self-motivated decision making and interactions in the game world. Thus, paratexts like walkthroughs, after-action reports and let's plays are discursive manifestations of individualized player experiences that generate and simultaneously individualize players' experiences of the "orthogame" (Carter et al. 2012) itself. Souvik Mukherjee (2015: 106) goes as far as to argue that, "to construct any textuality for the videogame, the paratextual needs to be considered first".

In this chapter we propose to take videogame paratextuality one decisive step further and argue that unplanned, spontaneous player communication during physically co-located 
gameplay, which we call co-situated (or "co-sit") player interaction, is a form of paratext as well, comparable in its immediacy and meaning-making negotiations for instance to the conversations between a parent and a child during a joint reading session. Indeed, co-sit gaming might be seen as one of the most intrinsic forms of metaludic communication as it happens in direct response to on-screen action and reflects the experiential aspects of videogames' mechanics and narrativity with a maximum degree of immediacy and emotional rigor.

Co-sit interaction can be differentiated from other, seemingly spontaneously occurring oral videogame paratexts such as let's plays. Both genres emerge synchronously during gameplay, and although let's plays do occasionally feature more than one player, they are essentially videos posted on channels like YouTube, while co-sits are essentially private gaming sessions and not primarily intended for publication. Surely, some players record their co-sit play and put it on the PlayStation network for example, but compared to performance-oriented and often (partly) scripted and rehearsed let's plays, co-sits tend to be unplanned and primarily socially rather than public-performance or even - in the case of professional YouTube channels - commercially oriented let's plays. Let's plays are also conceptually monologic, with (typically) one player commenting on their gameplay, whereas co-sits are conceptually dialogic, in response to screen action, bringing relationships between players to the fore. In other words, let's plays and other forms of public metagame paratexts are essentially performative in function, addressed to an audience of fans, followers, or followers-to-be, and the conative nature of let's plays is a lot more pronounced than the more competitive, inward-looking, interactional intent behind cosits.

\section{Subverting the discourse of power}


Johan Huizinga's (1950) concept of the magic circle has been widely debated and partly subverted by contemporary ludologists (e.g. Consalvo 2009). It describes play in terms of being in "a temporary world within the ordinary one" (Dippel and Fizek 2016: 1), a world that we enter when we embark on gameplay and that makes us feel immersed and separated from the actual world. The magic circle allows or even forces players to adopt what Bernard Suits (2005) has called a lusory attitude, a psychological state that makes players accept and embrace the rules of the game they are engaged in, including its embedded values and ethical implications, no matter how unethical those rules and conditions may be outside the magic circle. What this implies is that, while in the magic circle, players dispense with many of their actual-world rules and values and replace them with alternative value and rule sets.

Player groups conjoin the idea of lusory attitude with that of community of practice, which, according to cognitive anthropologists Jean Lave and Etienne Wenger (1991), refers to a group of people who share an interest, a craft, and/or a profession. The group can evolve naturally because of its members' common interest in a particular domain or area, or it can be created specifically with the goal of gaining knowledge related to their field. Importantly, these shared practices involve various social actions, including specific linguistic and communicative practices. Furthermore, the tacit knowledge shared by members of a specific community of practice is akin to, or contains within itself, the linguistic concept of pragmatic presupposition (Stalnaker 1974), which refers to the shared assumptions about the context in which communication takes place and about the interlocutor and their knowledge and world view. Habitual game players are fully aware of the shared world views and social practices afforded or even demanded by specific gaming situations, and while gameplay itself is understood in 
terms of a general fun- and entertainment-oriented activity, player expectations and social practices can vary widely depending on the game genre or even the game itself.

Previous work by Ensslin (2012) on player discourses has documented the frequent use of swearing and other types of strong language, which reflects highly specific social contexts and communicative embeddings. In Swearing in English, Tony McEnery (2006) argues that swearing subverts the discourse of power, a purist language ideology pursued by powerful social groups, who have the cultural capital (Bourdieu 1984) to determine what is appropriate language use for any given situational context. The discourse of power works hegemonically, i.e. it is reconfirmed and perpetuated by anybody following and adapting to it. We would argue that this is exactly what most gamers refuse to do: they subvert the discourse of power (in the sense of privileged, elitist social codes) by communicating in ways that are not considered "refined," that are regularly censored in pre-watershed public broadcasting, and typically scolded by powerful stakeholders such as parents, teachers, and politicians in the media.

Subverting the discourse of power happens at many levels of metaludic communication and player culture more generally:

player behaviors can often resist or even challenge the underlying social order [imposed by rules and subscribed by players to be able to play the game in the first place]. This includes technically specialized interventions, such as hacking and modding, as well as widespread player practices such as cheating, technological appropriations, subversive readings, interpersonal relationships, and the production of unofficial game "paratexts" (such as fan fiction, walkthroughs, etc.). (Grimes and Feenberg 2009: 108) 
Interestingly, however, as our ensuing data analysis will demonstrate, what may prima facie and plausibly be seen as subversive behavior is not necessarily understood as such by players themselves - at least not in their conscious, explicitly rendered perceptions.

\section{Player discourses}

In The Language of Gaming, Ensslin (2012) outlines three dominant player discourses that can be found across videogame paratexts: the Discourse of Fun, the Discourse of Cool and the Discourse of Appreciation. All three can be viewed under the broad umbrella of affective discourse because they all express emotions in more or less explicit ways: fun is inherent in the experience of play, although, of course, the game itself does not necessarily have to be funny or even evoking positive feelings (think of horror games); cool is a concept that paradoxically and effectively blends detachment and engagement, and appreciation involves evaluative discourses relating to game ratings and other forms of quality assessment.

In this chapter we align players' use of so-called Bad Language Expressions (“BLE," adapted from McEnery's more restrictive term, "Bad Language Words," 2006) with the Discourses of Fun ("DoF") and Cool ("DoC") in particular. The DoF reflects players' extreme emotional investment in the games they play and the activities they perform in and around gameplay. This emotional investment, combined with a strong dedication to ritualized performance, typically manifests in the form of a vast diversity of expressive interjections, including response cries (Conway 2013; Goffman 1981) and laughter. Interjections signaling enjoyment can adopt different emotional connotations, such as malicious and/or supportive amusement, awe, shock, or bawdiness.

The DoC is slightly more elusive and challenging to capture, but it generally revolves around the paradox of detached engagement: on the one hand cool is about projected emotional 
detachment whilst on the other it is about the performance of playing along as a mode of social engagement and emotional bonding. "Cool both enables and prevents relationships developing" (Nicholson and Hoye 2008: 28), and it is exactly this paradoxical tension that makes it so socially attractive. Put differently, cool detachment serves the purpose of trying to fit in and being accepted by others through respect rather than overt expressions of personal interest or emotional engagement. Cool assumes a sense of propriety, which involves knowing just how close one needs to get in order to both respect and gain respect from the other. The ideal cool distance cannot be guaranteed in advance, but it needs to be neither too far, nor too close, ensuring the other's retention of effort in the relationship as well as the potential benefits of what a good relationship with the other can offer (Blackshaw and Long 2005: 253). In player discourse, then, the DoC marks the playful interception between social and antisocial communication, such as mock bravado and instances of clipped lexis and very short, staccato syntax in players' turntaking, as well as an overuse of expletives and other forms of BLE.

\section{Methodology}

This particular study was motivated by the anecdotal observation that swearing in videogame paratexts, and especially spoken paratexts, is abundant. Our aim was therefore to find out more about (1) the actual patterns of BL use in player communication and, more specifically, spoken, synchronous turn-taking during co-sit play, and (2) how players themselves explain their linguistic behavior and, specifically, their motivations for using BL, following an actual co-sit session. For the first goal, we first defined a BLE tagset for corpus annotation, based on McEnery's (2006: 25; see also McEnery et al. 2006: 264) categories of "Bad Language Words" (BLW). McEnery distinguishes between actual expletives, or swear words related to sex, defecation, and other body parts and fluids generally known to trigger revulsion (FUCK, PISS, 
SHIT, CUNT, BLOODY), animal terms of abuse (PIG, COW, BITCH, ASS), sexist terms of abuse (BITCH, WHORE, SLUT), intellect-based (IDIOT, PRAT, IMBECILE, DORK), racist (PAKI, NIGGER, CHINK), religious (JESUS, GOD, HEAVEN, HELL, DAMN, HOLY) and homophobic (QUEER, FAG, POOF, DYKE) terms of abuse. There is a certain degree of overlap in some terms of abuse (e.g. BITCH and COW are both sexist and animal-related), and they often combine into expressions like HOLY SHIT.

Data collection took place in summer 2015 and resulted in a corpus of four thirty-minute video- and sound-recorded co-sit gaming sessions featuring undergraduate students (two per cosit session) at Bangor University, where both authors were based at the time. Six out of our eight participants were male (we did not follow a gender critical approach in this study and therefore followed a logistically feasible rather than gender-balanced recruitment strategy) and all participants were native $(\mathrm{n}=7)$ or near-native $(\mathrm{n}=1)$ speakers of British English. All participant pairs were gaming "buddies," i.e. they were habitual co-sit players and used to playing with each other. To further support their familiarity levels, they were allowed to play a game of their choice. The games they chose were Helldivers (Arrowhead Game Studio 2015; PS4 version; P1/P2), Streetfighter X Tekken (Capcom 2012; Windows with Fightstick; P3/P4), Halo 4 (343 Industries; Xbox 360; P5/P6), and Yoshi's Woolly World (Good-Feel 2015; Wii U; P7/P8). Each session was immediately followed by semi-structured interviews with each co-sit pair. The corpus contains 10,287 tokens. The transcripts were analyzed as raw data and subsequently tagged in terms of BLE types as outlined above. We then analyzed the corpus data in terms of frequency lists, keywords (using the BNC Spoken as reference corpus), concordances and keywords in context, collocations and clusters, using WordSmith 6.0. The interviews were coded for thematic 
nodes in NVivo 11 with a view to distilling some grounded theory relating to habits and motivations for bad language.

\section{Data and findings}

\subsection{Corpus analysis}

A look at the keyword lists of the co-sit transcripts strongly suggests overuse of the expletives SHIT $(0.21 \%$, keyness $=101.06$; used particularly often in the phrases OH SHIT and ARR SHIT, and directly collocated by religious BLEs such as OH GOD - see below), CRAP (0.12\%, keyness $=77.01)$, and FUCK $(0.07 \%$, keyness $=24.37$, also used as prepositional verb, FUCK OFF, and in the formulaic verb-complement phrase, FUCK ME) - and these values only reflect the represented core forms themselves, excluding derivatives such as CRAPPY (used for example in CRAPPY LITTLE ELF). Also frequent were religious BLE's (GOD, 0.45\%; keyness $=557.12$; used specifically in $\mathrm{OH}$ MY GOD and $\mathrm{OH}$ GOD and in combination with expletive BLEs - see above).

Overall, the distribution of BLEs across the thus annotated corpus is shown in figure 6.1, and their normalized frequencies in table 6.1. The vast majority of BLEs in the corpus are religious terms of abuse and actual expletives, and there is a small percentage of animal, intellectbased and sexist terms of abuse. Racist and homophobic BLEs are not represented in the current dataset.

(Insert fig. 6.1 here)

In addition to overusing ${ }^{1}$ certain BLE categories, however, players also overused certain terms of endearment and politeness, such as THANKS $(0.15 \%$, keyness $=58.52)$ and SORRY $(0.35 \%$, keyness $=70.06)$. A closer look at the concordance of THANKS reveals that it is often used cynically, as in THANKS, MATE or THANKS FOR THE REASSURANCE. The cynical 
reading of these phrases is reconfirmed by a multimodal analysis of the audiovisual data recordings, which took into account intonation and body language. Terms of gratitude were often used ironically with the intention to tease the other player about their antisocial behavior in the game, for example, or about an error they had just made via their player-character that had harmed either the speaker's player-character or both player-characters. Terms of apology, on the other hand, were commonly used sincerely, typically to exculpate the speaker from making a perceptually grave blunder (e.g. "Aww gosh, I'm really sorry. That was my fault there.”) In turn, the player at whom the apology was directed would often respond with phrases containing another overused lemma, FINE, as in "No, hum, it's fine. You carry on." Further reconfirming polite interaction is the fact that FINE was used more generally in utterances signaling mutual reassurance ("It's fine. You can do this" or “Are you all fine there?").

The above observation underlines previous findings made by Ensslin (2012) suggesting a mixture of emotional detachment and engagement, which is characteristic of the Discourse of Cool. Unsurprisingly, COOL itself was overused by participants $(0.08 \%$, keyness $=44.78)$, which is partly due to it frequently occurring in reduplicative clusters (COOL, COOL!). Examining the actual uses of COOL allows a more complex picture. In the following passage, the speaker's emotions in response to on-screen action switch rapidly between controlled "cool" to a panicky expletive, reflecting the realization that death was imminent for a split second. The end of the passage ("Right, okay") echoes the restoration of the speaker's self-control, or at least a level of acceptance that marks willingness and ability to move ahead in the game. This restoration of self-control can be understood in the context of aiming to keep one's "cool" in the game and its metaludic discourse, and thus to maintain positive rapport between the players.

P2 Cool. Oh shit, I almost got lazered by you then. Right, okay. 
The following exchange between $\mathrm{P} 1$ and $\mathrm{P} 2$ sees $\mathrm{P} 2$ announcing a rather inglorious yet somewhat gentlemanly choice of weapon, compared to the high-tech armor typically used in the Helldivers universe. The detached way in which P2 talks about how he will kill the final enemies without seemingly having to fear counterstrike or self-destruction is reflected in P1's question referring to P2's detached engagement in the termination process, and P2's ensuing unambiguously affirmative answer, "Yeah".

P2 I'm gonna end this mission with a pistol.

P1 Wow. Don't you feel cool? < giggles >

P2 Yeah.

\subsection{Interviews}

To gain a more profound understanding of participants' motivation for overusing BLEs, we conducted semi-structured interviews following their co-sit sessions. They were asked more generally about their linguistic habits during gameplay and particularly about how they accounted for the strong presence of bad language and expletives in particular in player discourse. One question related to McEnery's (2006) argument linking swearing to subversive behavior specifically asked them actually about subversive behavior, which none of them seemed to agree with, at least not in the sense of being the main motivation behind swearing in player discourse. ${ }^{2}$ Instead, they explained their BLE motivations and behavior in terms of the following hierarchical list:

(1) Immersion, spontaneity, control: participants explained that their strong levels of immersion in a game took away any linguistic inhibitions by which they would be impacted in other situations of everyday life; 
(2) Strong feelings, including frustration, anger, fear, freedom, emotional investedness in serious play, self-expectations and ambitions;

(3) Communities of practice and their imposition of copycat behavior and social norms, which cause players to apply certain medium- and context-specific social and linguistic behaviors as part of individual personality management;

(4) Metacommunication (Bateson 1972) commanding players to exhibit certain degrees of fake emotions, irony, and sarcasm;

(5) Taboo removal, specifically in relation and opposition to norms followed and imposed by older generations and culture-specific norms;

(6) Player, platform and game specificity, i.e. specific linguistic choices such as the amount and selection of BLE is contingent on co-player, platform, or even the game itself.

(7) Behavior linked to online culture, which tends to give players a sense of safety and privacy regarding their social and linguistic behavior but also generates the need to express feelings more strongly through verbal means than in face-to-face communication.

Blending motivations (1) and (2), one participant pair described the immersion-related removal of inhibition in very material terms, as a profanity filter that evaporates in an emotionally heightened, immersive state (Pn refers to participant number 1-8; R refers to the interviewing researcher; // marks partial overlap with next speaker's turn; <.> marks one-second pause; <..> marks pause of two to three seconds ${ }^{3}$ ):

P1: $\quad$...you get so immersed

P2: $\quad$ Yeah you get kind of immersed 
P1: $\quad$ Because it's like ah, shit, if we die we've failed//

P2: $\quad$ You get excited and yeah you feel like it's gonna be like a failure if you er if you mess up so <.> I don't know, you kind of just lose your filter a little bit I guess. $<. .>$ The profanity filter just sort of//

\section{P1: $\quad$ It evaporates $<$ giggles $>$}

When referring to community of practice related behavior, another participant pair expressed themselves thus:

P3: $\quad$ But yeah, I'd say that, it's a more relaxed community.

P4: Yeah.

P3: Gamers in general. Er, I don't think there's so much hang-up on politeness, erm, $<_{. .}>$yeah like $<_{\text {. }}>$

P4: $\quad$ There's almost like a desensitization to swearing in general, like it doesn't hold the same weight, like the words don't hold the weight that they usually mean or or do with other people, I think, within the circle of people playing games.

Thus, players' community of practice is described as less restrictive and socially rule governed than other communities surrounding P3 and P4. Furthermore, expletives seem to be so common in players' community of practice that members have become "desensitized" to the social taboos associated with them in other communities and situations of everyday life.

Participant utterances represented in the following passage relate to metacommunication in play, which refers to Bateson's (1972) observation that play behavior, and particularly playfighting, communicates its simulative nature, involving "deception, threat, and imitation" 
(Mitchell 1991: 73). This is reflected in P1's description of trying to come to terms with frustration that, if seen rationally, may not be legitimized by the "joke" nature of the game because players are bound to "deluded" into being overly affected emotionally. In other words, rationality should tell them not to get emotionally involved in the fictional interactions represented in and by gameplay. At the same time, however, play is perceived as extremely serious and real ("the stakes are so high"), which gives rise to exclamatory expressions of frustration or anger in moments of failure, such as "arr, fuck" and other expletives:

P1: I think it's because in the moment, so when you mess something up, like, you can vent your frustration although you're like you're not really frustrated but you kinda like oh well first it's only a joke, isn't it, and then "arr, fuck." <last two words whispered>

P2: $\quad$ Yeah it's sort of like obviously, ha, we're not deluded, we know it's a a game but like in your head, when you're playing, it kind of like the stakes are so high.

Incidentally, the fact that the quoted expletives at the end of P1's turn are whispered rather than spoken at the regular volume of interaction signals that the social context of the interview is perceived as too formal to utter the expletives in the same way he would in a gaming context. This underlines the discrepancy in perceived appropriateness between both communicative situations.

The topic of taboo removal linked to generational etiquette changes and culturespecificity was mentioned in particular by participants 3 and 4 (who had been playing Streetfighter IV): 
P4 One of [the reasons] is the generation that like sort of is the main players of videogames like it's normally the younger generation, if you don't have that sort of built-in taboo reservation for swearing

P3 Yeah, I think it's generational because I erm I did spend a year in France, for a year, and I found myself like learning the swearwords in that language and using them fairly frequently and then people would come up to me and say "Why do you swear so much?", and erm

$R \quad$ What kind of people would ask you that?

P3 I was at university over there.

$R \quad$ Okay.

P3 So students about the same age as me. They were just picking me up for swearing so much because I assumed they'd do it at the same frequency as people in English.

The fact that the above passages refer to swearing in situations other than videogames provides further context for the dominance of BLE in player communication. However, the use of BLE in terms of intensity, frequency, and/or linguistic choices can also be linked to the specific game or game genre being played, the platform being used, or the types of games produced by specific companies and the designs they stand for:

P8 Yeah it's sort of like a control thing.

$R \quad$ Would you say that is true, does that make sense? 
P8 For the most part yeah, especially when it comes to like it depends like console to console as well. ... So it's stuff like Sony and Microsoft, you will get a lot of people like they will play the games and they will swear a lot but if you're like Nintendo, you won't get it as much.

R Uhum.

P8 Like it depends on like, the different companies like how their games are and like if you've got a bright, colourful game you don't really wanna be like really like rude, do you?

R Uhum.

P8 But if you've got something like a war game or something, you're gonna be like, you're gonna get the emotions getting angry and stuff like because it just reflects how the games are.

Interestingly, here P8 (who played Nintendo's Yoshi's Woolly World in the preceding cosit session, links the use of BLEs to a need to express control, or power, in relation to their abilities or experience with a specific technology - a console or type of game. Yet, predictably, BLE habits can also vary massively from player to player, as P5 (one of the Halo 4 players) confirmed in his interview: "I don't usually swear at all," and in whose co-sit transcript there really was no instance of expletives to be found, and only a few instances of religious BLEs.

Finally, participants related the frequent use of BLEs to online identity management:

P4 I think a big part of [BLE use] comes as well from the online aspect of not being there in the same room so the best way to like show your expression is sometimes like to overreact and that means like to swear you head off all the time. 
Finally, the above-mentioned corpus data pointing at somewhat paradoxical polite behavior mixing with the use of BLEs were complemented by interviewees explaining that apologetic or other types of polite behavior were indeed quite common in their co-sit communication:

R: $\quad$ And what struck me ..., you apologized quite a lot as well.

P7: Yeah, like “sorry, I just ate you." < all laugh>

P7: "Sorry to carry you around as an egg now."

P8 <laughs $>$ Just not normal phrases but in the context of the videogames it is.

$R \quad$ Okay, and would this be quite typical, like is this usual with you more or less?

P7 Yeah//

P8 Yeah.

Importantly, polite expressions are here ridiculed or parodied as they are combined with fictional lexis relating to the fantasy world of Yoshi's Woolly World. An important motivation for polite behavior in heated and often verbally abusive player communication is given by P1 and P2 (the Helldivers players):

P2 I think we also tend sort of er <.> I can't think of a better phrase to describe it, so like "bro-up"

P1 Bro-up, yeah.

$R \quad$ Bro-up?

P2 Yeah// 
P1 Yeah.

P2 Like stick together sort of thing.

P1 Yeah like

P2 We'll we'll scate, scathe each other but ... [it] pretty much adds to the humour for us though.

$R \quad$ Yeah I thought that was really interesting because you were using a lot of polite phrases as well. You would say like "thank you” or “you're welcome," or you would apologize or something, and then all of a sudden there was a swear word like "shit" like.

P1 <laughs $>$ That's pretty much what we do <laughs hysterically $>$

$<$ All laugh $>$

P1 It's like oh, we're professional, er. <laughs>

Thus, the need to "bro-up" and to create an atmosphere relaxed and amused enough for both BLEs and polite language to contribute to fun and laughter seems paramount to these and other co-sit players.

\section{Discussion}

Our corpus analysis showed that certain types of BLEs were overused in the co-sit player interactions we studied in this project. Particularly dominant were religious BLEs and actual expletives, with phrases containing GOD, SHIT and FUCK featuring particularly high keyness values. This finding correlates with Ensslin's (2012: 94-95) finding that, generally speaking, expressive speech acts are dominant in player discourse, signaling the need to communicate a 
variety of feelings and emotional attitudes, such as joy, pain, desire, pleasure and sorrow. When immersed in gameplay, players become emotionally involved as they need to re-prioritize their aims and objectives in order to succeed in the gameworld. Success and failure lie close together and tend to be represented in terms of extreme in-game metaphors such as, quite literally, life and death. Players' emotional responses to in-game events and circumstances therefore tend to be just as, if not more heightened than actual life events, and these emotional outbursts manifest themselves in a variety of linguistic and paralinguistic expressions,such as spill cries (spontaneous, impulsive expressions of negative surprise or anger, typically after having (been) flawed in or by a game), threat startles (controlled expressions of negative surprise or fear), revulsion sounds and laughter (Conway 2013). The boundary between spill cries and threat startles is of course a fluid one as the extent to which an affective speech act is uttered in an impulsive or controlled fashion is not only subjective to speaker and listener but also a matter of degree rather than an absolute distinction. It can be argued, however, that fully verbalized expressive speech acts like OH GOD or FOR FUCK'S SAKE may indicate a more controlled (threat-startle) response than non-verbal interjections like "WHOOA!"

The predominance of religious BLEs in our corpus may be a reflection of the fact that "religious swearing [in English] is not as powerful as it once was - linguistic functions that once were performed by words and phrases that called on God or blasphemed him are now performed mostly by words for taboo human body parts and actions" (Mohr 2013: 10). Thus, religious BLE's have lost much of their original taboo stigma and are used commonly, not only in player discourse, but everyday verbal interaction more generally. Conversely, the almost complete absence of other types of BLEs, namely animal, sexist, and intellect-based BLEs may point to either personal preferences on the part of the admittedly small population under investigation 
here, or indeed the fact that they reflect a more general convention followed by (British) players' community of practice.

To further explain the observed patterns of BLE use in our corpus, our interview data suggested that the frequent use of expletives in player discourse is not only a reflection of the heightened and authentically felt emotions experienced by immersed players. A number of responses indicated that players' communities of practice operate according to different, more relaxed social norms than other communities surrounding our participants. Interestingly, participants did not link the community of practice motivation with a need to revolt against the discourse of power (McEnery 2006) but rather with the social safety offered by the magic circle (Huizinga 1950), which endorses the suspension of everyday social rules within its own psychological and ludonarrative confines. They indicated that the relaxed attitude towards profanity may further be related to generational shifts in assumptions about appropriate and inappropriate verbal behavior, which again reflects a general trend among young people to use BLEs including expletives frequently and habitually as part of their peer group's accepted if not mandatory code of communication, and specifically codes dictated and/or perpetuated by computer-mediated communication (Kiesler et al. 1984; Baron 2003).

Generally speaking, the range of motivations offered by our participants points at a complex and relativistic motivational framework for BLE use in gameplay. They mentioned the importance of metacommunicative play (Bateson 1972), which renders players' awareness of the fictional nature of their ludic interactions and indexes the importance of expressing the simulative nature of play through ludic behavior that marks itself as self-consciously ludic. The anonymity yielded by online culture was another contextualizing facture mentioned by participants, which adds an interesting virtual component to the physicality of co-situated 
gaming. Players seem to retain an awareness of the hypermediated landscape in which their specific co-sit experiences are embedded, thus leading to attitudes and assumptions that cross over from online to physically co-experienced communication - bearing in mind the increasingly fluid boundaries between the former and the latter. Finally, participants demonstrated a keen awareness of the platform-, genre-, game- and brand-specificity that their communicative behavior is contingent upon, as well as the ways in which they adjust their verbal choices to individual co-players.

Our data further indicates that co-sit discourse is altrocentrically oriented and emerges in continuous mutual responses to what the other player has done or said vis-à-vis on-screen action, rather than being directed at the player's egocentric performance in let's play discourse, for example (see Ensslin 2016). We found that co-sit players in our study combined forms of verbal abuse and aggression with distinct and frequent forms of politeness (especially P1/2 and P7/8). There was a lot of polite discourse involving reciprocal thanking, apologizing, and reassuranceseeking between players, an mechanism described by one player pair as "bro-up" procedure. Importantly, this bro-up mechanism was perceived to increase the humorous side of co-sit gameplay, which in turn underscores the multi-layered nature and social significance of the Discourse of Fun in gameplay.

What is striking about co-sit interaction is the extreme affective polarity of players' expressed emotional states and the frequent and immediate shifts between extreme forms of affective discourse to rational composure. Thus, it can be argued that the discourse of co-sits follows important social norms related to detached engagement, or dictates of the Discourse of Cool. It also became clear, however, that in each pairing there was a hierarchy established by the cultural capital (Bourdieu 1985) of the stronger or more experienced gamer. This hierarchical 
relationship manifested itself in mechanisms of asking for and giving advice, but also in patterns of ridicule and directive speech acts.

\section{Conclusions}

This chapter offered an examination of co-sit player communication and focused in particular on patterns of BLE use and on what might be perceived as its paradoxical counterpart: politeness in otherwise heated and seemingly emotionally uncontrolled player communication. We found that this apparent paradox is subverted by the important context-specific ways in which the use of BLE can be seen as part of a community-of-practice-intrinsic code which contributes to perceived levels of fun and social bonding as it is often used humorously and with the aim to increase psychological connectedness between players. In our dataset, there was a general pattern that religious and expletive terms by far outweighed other types of bad language, and that specific BLEs are key in the sense of being overused compared to common everyday oral interaction. Our corpus findings were relativized and contextualized through data garnered from participant interviews, which yielded a complex, multi-layered and relativist picture of the motivations behind BLE use and specifically swearing in (British English) co-sit discourse.

Clearly, the limited scope of this study cannot yield any statistically significant or generalizable results. Nor can the results gained from a spoken corpus that is just over 10,000 tokens in size tell a conclusive story beyond the data set investigated at the time, and in its sitespecific context. It may further be conceded that participant behavior and responses will likely have been affected by the Hawthorne effect, as the researchers were present during the videorecorded co-sit sessions in order to be able to refer to specific verbal behavior in the directly ensuing interviews. Nonetheless, the findings strongly corroborate anecdotal evidence of player behavior experienced frequently by both authors of this paper. 
By ways of a final thought, the immediate communicative and spontaneously emergent nature of co-sits begs the question of whether co-sit discourse is indeed paratextual or whether it should rather be regarded as part of the actual text, or orthogame, itself (Carter et al. 2012). After all, game narrative does not emerge until players perform in-game interactions, and the conversations between co-sit players are at least partly a manifestation of the first-person enactment of their embodied roles as avatars. Players often speak in-character, and their fictional dialogues alternates with metaludic dialogic commentary and response cries. This embodied enactment performs an important act of dual situatedness, thus corroborating social bonding and legitimizing verbal and other forms of abuse. Future research is clearly needed that will refine our understanding not only of male-identified "bro-up" sociality in co-sit gameplay, but, importantly, that will cast a far more gender-inclusive picture of quasi-paradoxical cooperative ludic behavior.

\section{References}

1. Industries (2012), Halo 4, Redmond, WA: Microsoft Studios.

2. Arrowhead Game Studios (2015), Helldivers, San Mateo (CA): Sony Interactive Entertainment.

3. Aylett, R. (1999), 'Narrative in virtual environments: towards emergent narrative', in Proceedings of AAAI Symposium on Narrative Intelligence, pp. 83-86.

4. Baron, Naomi S. (2003), 'Language of the internet', in A. Farghali (ed), The Stanford Handbook For Language Engineers, 1-63. Stanford, CA: CSLI Publications.

5. Bateson, G. (1955), A theory of play and fantasy, Psychiatric Research Reports, II: 177-178. 
6. Blackshaw, T. and J. Long (2005), 'What's the big idea? A critical exploration of the concept of social capital and its incorporation into leisure policy discourse', Leisure Studies, 24: 239-58.

7. Bousfield, D. (2008), Impoliteness in Interaction, Amsterdam: John Benjamins.

8. Capcom (2012), Streetfighter X Tekken, Osaka: Capcom.

9. Carter, M., M. Gibbs and M. Harrop (2012), 'Metagames, paragames and orthogames: A new vocabulary', Proceedings of the International Conference on the foundations of Digital Games. ay 29-June 1, Raleigh, NC, USA, ACM.

10. Consalvo, M. (2009), Cheating: Gaining Advantage in Videogames, Cambridge, MA: MIT Press.

11. Conway, S. (2013), 'Argh! An exploration of the response cries of digital game players'. Journal of Gaming and Virtual Worlds, 5 (2): 131-146.

12. Dippel, A. and S. Fizek (2016), 'Ludification of work or Labourisation of play? On workplay interferences', in Proceedings of the $1^{\text {st }}$ Joint Conference of DiGRA and FDG, https://library.med.utah.edu/e-channel/wp-content/uploads/2017/02/paper_1681.pdf.

13. Ensslin, A. (2012), The Language of Gaming, Basingstoke: Palgrave Macmillan.

14. Ensslin, A. (2016), 'The language of gaming: Affective discourse patterns in two videogame paratext genres'. Keynote, LEXESP 2016: Videogames and Language, May 5-6, 2016, University of Alicante.

15. Genette, G. (1991), 'Introduction to the paratext', New Literary History, 22: 261-272.

16. Goffman, E. (1981), Forms of Talk, Oxford: Blackwell.

17. Good-Feel (2015), Yoshi's Woolly World, Kyoto: Nintendo. 
18. Gray, J. (2010), Show Sold Separately: Promos, Spoilers, and Other Media Paratexts, New York: New York University Press.

19. Grimes, S. M. and A. Feenberg (2009), 'Rationalizing play: A critical theory of digital gaming', The Information Society, 25: 105-118.

20. Huizinga, J. (1950), Homo ludens, Boston, MA: Beacon Press.

22. Jenkins, H. (2004), 'Game design as narrative architecture', in N. Wardrip-Fruin and P. Harrigan (eds), First person: new Media As Story, Performance, And Game, 118-130. Cambridge, MA: MIT Press.

21. Kiesler, S., J. Siegel and T. W. McGuire (1984), 'Social psychological aspects of computer-mediated communication', American Psychologist, 39: 1123-1134.

23. Lave, J. and E. Wenger (1991), Situated Learning: Legitimate Peripheral Participation, Cambridge: Cambridge University Press.

24. McEnery, T. (2006), Swearing in English: Bad Language, Purity and Power from 1586 to the Present, London: Routledge.

25. McEnery, T., R. Xiao and Y. Tono (2006), Corpus-Based Language Studies: An Advanced Resource Book, London: Routledge.

26. Mitchell, R.W., 1991, 'Bateson's concept of 'metacommunication' in play', New ideas in Psychology, 9: 73-87.

27. Mohr, M. (2013), Holy Sh*t: A Brief History of Swearing, Oxford: Oxford University Press.

28. Mukherjee, S. (2015), Video Games and Storytelling: Reading Games and Playing Books, Basingstoke: Palgrave Macmillan. 
29. Nicholson, M. and Hoye, R. (2008), Sport and Social Capital, Burlington, MA:

Butterworth-Heinemann.

30. Ryan, M.L. (2004), Narrative Across Media, Lincoln, NE: University of Nebraska Press.

31. Stalnaker, R. (1974), 'Pragmatic presuppositions', in Munitz, M. and P. Unger (eds),

Semantics and Philosophy, 197-214, New York: New York University Press.

32. Suits, B. (2005), The Grasshopper: Games, Life and Utopia, Peterborough, ONT:

Broadview Press.

Figure 6.1. Relative distribution of BLEs across corpus

Table 6.1 Normalized frequencies of BLE categories with types occurring in corpus

\begin{tabular}{|c|c|c|c|}
\hline Category of BLE & Types & $\begin{array}{l}\text { Frequency (normalized) } \\
\text { relative to entire corpus }\end{array}$ & $\begin{array}{l}\text { Percentage } \\
\text { of all BLEs }\end{array}$ \\
\hline $\begin{array}{l}\text { rel (religious terms of } \\
\text { abuse) }\end{array}$ & $\begin{array}{l}\text { GOD, JESUS (CHRIST), } \\
\text { JEEZ, DARN, (BLOODY) } \\
\text { HELL, GODDAMMIT, } \\
\text { HOLY LORD }\end{array}$ & 0.00651 & $49 \%$ \\
\hline $\begin{array}{l}\text { swear (expletive), } \\
\text { including euphemisms }\end{array}$ & $\begin{array}{l}\text { CRAP, CRAPPY, FUCK } \\
\text { (OFF), } \\
\text { FECKING, ASS, SUGARIN(G), } \\
\text { BLOODY (HELL), SHIT, } \\
\text { SHITE, BOLLOCKS, BUM }\end{array}$ & 0.00612 & $46 \%$ \\
\hline $\begin{array}{l}\text { an (animal terms of } \\
\text { abuse) }\end{array}$ & (SON OF A) BITCH & 0.00029 & $2 \%$ \\
\hline
\end{tabular}




\begin{tabular}{|c|c|c|c|}
\hline Category of BLE & Types & $\begin{array}{l}\text { Frequency (normalized) } \\
\text { relative to entire corpus }\end{array}$ & $\begin{array}{l}\text { Percentage } \\
\text { of all BLEs }\end{array}$ \\
\hline sexist (terms of abuse) & (SON OF A) BITCH & 0.00029 & $2 \%$ \\
\hline $\begin{array}{l}\text { int (intellect-based } \\
\text { terms of abuse) }\end{array}$ & TWIT & 0.00019 & $1 \%$ \\
\hline ALL & & $1.33 \%$ & $100 \%$ \\
\hline
\end{tabular}

${ }^{1}$ We use the term "overuse" in relation to the reference corpus, the BNC Spoken.

${ }^{2}$ Interestingly, some participants mentioned taboo removal as a motivation for swearing (see point 5 in the ensuing list). Hence, whilst they all seemed to agree that their swearing behavior was not subversive per se, they aligned it with a generational shift that they considered to be the sociolinguistic context within which a more general increase in swearing behavior has to be seen.

${ }^{3}$ The oral discourse annotation conventions were adapted from Bousfield (2008: 8) 